\title{
The impact of contact pressure and road surface character to sliding friction coefficient
}

\author{
Hong-guo $\mathrm{Xu}^{1, \mathrm{a}}$, Bin Ma ${ }^{1, \mathrm{~b}}$, Cheng Zeng ${ }^{2}$ and Yan $\mathrm{Xu}^{3}$ \\ 'Jilin University, Changchun 130022, China \\ ${ }^{2}$ Research Institute of Highway ministry transport, Beijing 10088, China \\ ${ }^{3}$ Changchun Automobile Industry Institute, Changchun 130000, China \\ axhg335@163.com, ${ }^{b}$ mabin11@mails.jlu.edu.cn
}

\begin{abstract}
Keywords: Tire; Sliding friction coefficient; Road surface character; actual contact model.
Abstract. A improved sliding friction coefficient model is presented based on road surface character and rubber character to analyze how the road character, tire and contact pressure affect the sliding friction coefficient. Improved the sliding friction coefficient model by introducing the theory of true contact with rough road and combined with the simplified rubber viscosity. Verifying the efficiency of the improved model through comparison with the dynamic friction coefficient of Savkoor. Analyzed the impact of contact pressure to the actual contact area and the contact pressure, and how tire and road surface character influence the sliding friction coefficient. The simulation result shows that the improved model can precisely analyze the impact of contact pressure,road surface character and tire to the sliding friction coefficient.
\end{abstract}

\section{Introduction}

Studies have shown that[1],the factor affect the tire wear including the tire, road surface character, tire adhesion and the contact pressure etc. Considerable work has been carried out about the studies of tire imprinted, Frank W took the real vehicle test to research the relation between tires, road surface and tire brake imprinted[2]. In rough surfaces contact, Giuseppe Carbone proposed a GW improved contact model[3] based on the GW contact model and simplify the contact theory. In rubber sliding friction, G. Heinrich studied the rough rubber friction model for self-affine road[4]. At present it has not be seen the reports of systematic studies how the road surface character and the contact pressure impact to the tire explicit-implicit character. Therefore, it is needed to propose a improved sliding friction coefficient model to provide a theoretical to systematic analysis of the explicit-implicit character of tire prints.

\section{Theory of tire contact with the rough road}

Mechanism of tire to rough road.Many studies have shown that general road has the strong anisotropy in horizontal and vertical direction and the fractal theory can accurately characterize the self-affine fractal features of rough road,so the fractal dimension $D$ can be used to characterize the road fractal character within the scope of the level cut-off length ${ }^{\lambda}$ and vertical cut-off length $\lambda_{\perp}$. The fractal dimension $D$ is given by

$$
D=3-H
$$

Where: $H$ is Hurst's index.

Beyond the horizontal and vertical cut-off length, the surface can be seen as flat and then not character as self-affine surfaces. Mechanism of tire to rough road is shown in fig. 1.

Road parameters can be calculated with the height-difference correlation function $C(\lambda)$, it is given by 
$C(\lambda)=\lambda_{\perp}^{2}\left(\frac{\lambda}{\lambda_{\|}}\right)^{2 H}, \quad \lambda_{\min }<\lambda<\lambda_{\|}$

where: $\lambda$ is the wave length; $\lambda_{\text {min }}$ is the minimum wave length.

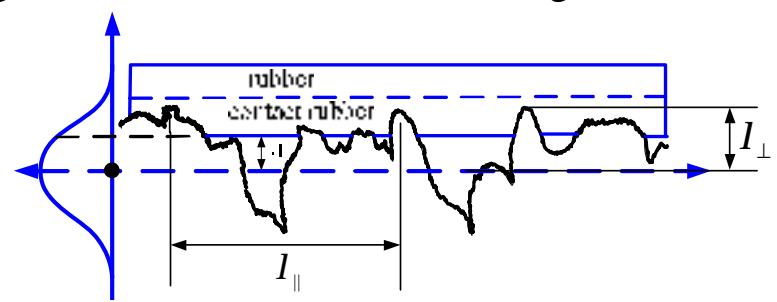

Fig. 1 The schematic of rough pavement cut-off length

When the tire and road contact in sliding state, the frequency power spectrum density in the frequency space is used to express the incentives of road to the tire, the height-difference correlation function can be converted into the frequency space within the self-affine range by fourier transformation, it is can be computed from

$$
S(\omega)=H \lambda_{\perp}^{2} \lambda_{1}\left(\frac{\omega}{\omega_{\min }}\right)^{2 D-7}, \omega_{\min }<\omega<\omega_{\max }
$$

Where: $\omega=1 / \lambda_{\text {is }}$ the space frequency; $\omega_{\min }=1 / \lambda_{\|}$is the minimum frequency power spectrum density; $\omega_{\max }=1 / \lambda_{\min }$ is the maximum frequency power spectrum density.

Actual contact theory. Tire and rough road not complete contact when they interaction, the nominal contact area is compose with the contact spots of road rough asperities and rubber surface. The actual contact model is shown in Fig.2.

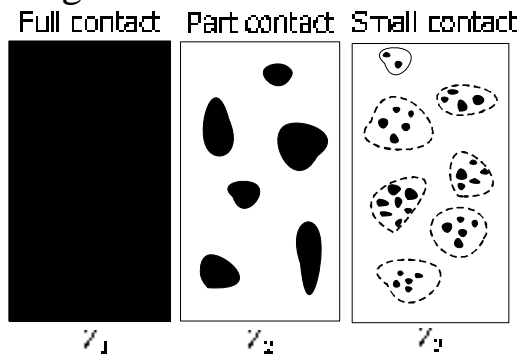

Fig. 2 Rubber partly contacting rough road at different amplification

Fig. 2 shows, in fractal surfaces with the contact pressure increasing the contact area increases as the rubber penetration depth increased. The relation between the contact pressure $F$ and the true contact area $A_{c}$ based on the GW improved contact model is

$$
F=\frac{E}{1-v^{2}}\left(\frac{m_{2}}{\pi}\right)^{1 / 2} A_{c}
$$

Where: $E$ is the young modulus of tread rubber; $v$ is the Poisson's ratio of tread rubber; $m_{2}$ is the moments of road space power spectrum. The $m_{2}$ is computed from

$$
m_{2}=\int_{\omega_{\min }}^{\omega_{\max }} S(\omega) \omega^{2} d \omega
$$


When interaction of tire to rough road, the relation of the true contact area $A_{c}$ and nominal contact area $A_{0}$ is

$$
A_{c}=\frac{1}{4} A_{0} \exp \left(\frac{t}{\sqrt{2}}\right)
$$

Where $t$ is the normalized distance, it is computed from

$$
t=d / \delta_{H D}
$$

Where: $d$ is the distance between the lowest rubber level and the mean profile height; $\delta_{H D}$ is the standard deviations of the height distribution.

Meanwhile, $t$ is the function of $\sigma_{0}$ based on the GW improved contact model, the $\sigma_{0}$ is computed from

$$
\sigma_{0}=\frac{\sqrt{2} E}{3\left(1-v^{2}\right) \pi}\left(\frac{m_{2}}{\pi}\right)^{1 / 2} \exp \left(-\frac{t^{2}}{2}\right) t^{-1} \Gamma\left(\frac{5}{2}\right)
$$

Therefore, the true contact area is not only related to the nominal contact area, but also the contact pressure, road character and rubber properties.

Simplified the viscoelastic energy loss.According to the expansion of Rolls-theory[5], when $p=1$, the incentive model is Rolls-mode; If assume each parallel Maxcell's unit mode is action at Rolls-mode, then all modes can be seen as the independent role at the Rolls-mode, and then $p_{i}=1, i=1,2,3 \ldots$.

For a single Maxcell model, the constitutive equations of dynamic stress response of viscoelastic materials is

$$
\delta(t)=\left[E(\omega)+i E^{\prime \prime}(\omega)\right] \varepsilon(t)
$$

For the simplified Maxcell-Weichert model, the constitutive equations of dynamic stress response of viscoelastic materials is

$$
\sum_{i=0}^{n} \dot{\delta}_{i}(t)=\sum_{i=0}^{n}\left[E_{i}(\omega)+i E_{i}^{\prime \prime}(\omega)\right] \varepsilon_{i}(t)
$$

Then

$$
E^{\prime \prime}(\omega)=\sum_{i=0}^{n} \frac{E \tau_{i} \omega}{1+\tau_{i}^{2} \omega^{2}}=\frac{n E \tau \omega}{1+\tau^{2} \omega^{2}}
$$

Make $b=\tau, a=n E \tau$, use $d$ to correction the data, then

$$
E^{\prime \prime}(\omega)=\frac{\omega a}{\left(d+\omega^{2} b^{2}\right)}
$$

Now, the unknown parameter is only the $\tau, E$. We can through the simple static experiments and numerical optimization to obtain a more accurate value $\tau$.For elastic modulus $E$, it can be calculated 
through the value get from the simple shore hardness meter. The viscoelastic energy calculation is simple after improved.

\section{Improved sliding friction coefficient model}

Sliding friction coefficient model.The sliding friction force combine with rubber properties and road character can be divided into two components[3,6-7]:hysteresis friction force $F_{H y s}$ and adhesion friction force $F_{\text {Adh }}$.

$$
\mu_{S}=F_{H y s} / F_{z}+F_{A d h} / F_{z}=\mu_{H y s}+\mu_{A d h}
$$

Where: $\mu_{H y s}$ is the hysteresis friction coefficient; $\mu_{A d h}$ is the adhesion friction coefficient; $F_{z}$ is the vertical load.

Combining with the GW improved contact model and the simplified viscoelastic energy loss, the hysteresis friction coefficient and adhesion friction coefficient can be calculated as.

$$
\mu_{s}=\frac{\langle\delta\rangle}{2 \sigma_{0} V}\left(\int_{\omega_{\min }}^{\omega_{\max }} \omega E^{\prime \prime}(\omega) S(\omega) d \omega\right)+\frac{\tau_{s} A_{c}}{\sigma_{0} A_{0}}
$$

Where: $\langle\delta\rangle$ is the excitated rubber layer thickness; ${ }^{\prime \prime}(\omega)$ is the rubber loss modulus; ${ }^{\tau_{s}}$ is the shear stress; $V$ is the rubber slip velocity;

Contrast verification.Contrast verification with the modified sliding friction coefficient[8] of Savkoor. When the tire is working on braking condition, the sliding friction coefficient of Savkoor is

$$
\mu_{s}=\mu_{x s}+\left(\mu_{x 0}-\mu_{x s}\right) \cdot \exp \left\{-\mu_{x}^{2} \lg ^{2}\left(\left|V / v_{x m}\right| \exp \left(-\left|V / v_{x m}\right|\right)\right)\right\}
$$

Where: $\mu_{x 0}$ is the static friction coefficient; $\mu_{x s}$ is the sliding friction coefficient; $\mu_{x}, v_{x m}$ is the friction parameters; $V$ is the slip velocity.

The contact pressure has an impact on sliding friction coefficient, contrast verification at the typical contact pressure of $0.3 \mathrm{MPa}$ for the removal of the contact pressure on the friction coefficient. The simulation data is listed in table 1 , the simulation results are shown in fig. 3 .

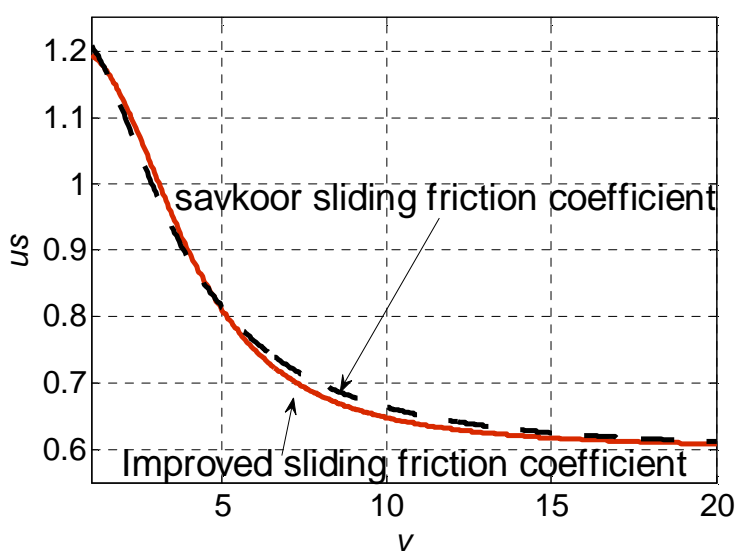

Fig.3 Comparison of the improved model with Savkoor friction coefficient

Table 1 Fitted values of friction coefficient

\begin{tabular}{ccccc}
\hline \multicolumn{5}{c}{ Savkoor parameter } \\
\hline symbol & $\mu_{x 0}$ & $\mu_{x s}$ & $\mu_{x}$ & $v_{x m}$ \\
value & 1.1 & 0.5 & 1.32 & 1.1 \\
\hline
\end{tabular}


From fig.3 it can see, contrast through the friction coefficient the simulation error between improved sliding friction coefficient model and sliding friction coefficient model of Savkoor is less than $3 \%$. So the improved sliding friction coefficient model able to explain the friction coefficient changes with speed and also consider the impact of road surface character and the rubber character. Meanwhile, the improved sliding friction coefficient model also provides a theoretical basis for systematic analysis of the tire mark hidden features.

\section{Simulation and results analysis}

Analysis of the true contact area simulation. Profiles were taken with stylus measurements for granite, and white light interferometry for asphalt respectively, the road fractal dimension is the slop of $C_{i}(\lambda)$, the typical granite and asphalt surfaces affine parameters are listed in table 2.

Table 2 Road affine parameter

\begin{tabular}{ccc}
\hline Road parameter & Granite & Asphalt \\
\hline $\mathrm{D}$ & 2.37 & 2.39 \\
$\lambda \perp[\mu \mathrm{m}]$ & 310 & 430 \\
$\lambda \|[\mu \mathrm{m}]$ & 2490 & 1440 \\
\hline
\end{tabular}

Road surface fractal character, contact pressure, tread rubber character will affect the size of the normalized distances[4]. For both road surface, use the 195/65R15 radial tire with the shore hardness $60 \sim 65$,stress at definite elongation is at 9 12MPa, tensile-strength $20 \mathrm{MPa}$ to progress the simulation test, the relation between contact pressure and the normalized distances is shown in fig. 4 .

Take the typical tire contact area $0.04 \mathrm{~m}^{2}$ to progress the simulation test, the relation between the normalized distances and the rough road true contact area is shown in fig.5.

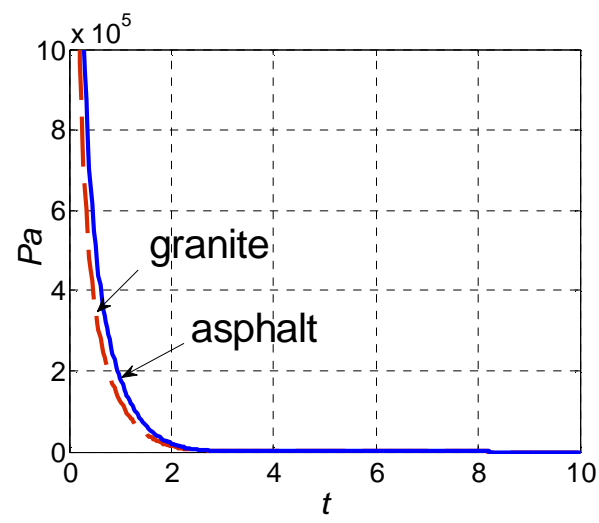

Fig.4 Relation between the normalized distances and the contact pressure

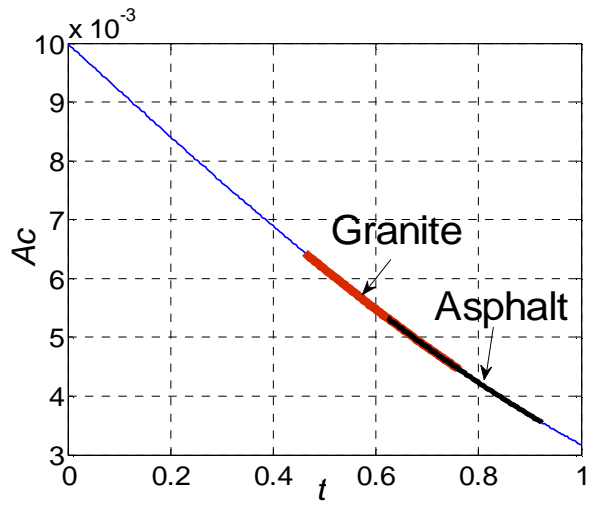

Fig.5 Relation between the normalized distances and the true contact area

Fig. 4 shows that in the same contact pressure, the normalized distances of granite is small than asphalt. Fig. 5 shows that the relation between the rough road true contact area $A_{c}$ and the normalized distances $t$ is approximately linear, The simulation results is consistent with the Roux[9] et al.

For the wave lengths of the granite road is small, the density of profile bigger than asphalt, the vertical cut-off length is smaller but the level cut-off length is bigger than asphalt, and the contact spots is more than asphalt, therefore the true contact area of granite is bigger than asphalt. Meanwhile, for the contact spots of granite is more than asphalt, so the contact area increase and hinder the profile penetration into the rubber, therefore the normalized distances is smaller, it is as shown in fig. 5. For two road, with the normalized distances increase the true contact area decrease, when the normalized distances is so bigger then the tire and road is separate the contact pressure is 0 . 
Analysis of the friction coefficient simulation.The typical contact pressure between the tire and road is $0.3 \mathrm{MPa}$, in the range of the vehicle vertical load, contact pressure change in the range of $0.2 \sim 0.4 \mathrm{MPa}$. So simulation is within the range of $0.2 \sim 0.4 \mathrm{MPa}$, road affine parameters is in table 2 , the results of simulation are shown in fig.6-fig.7.

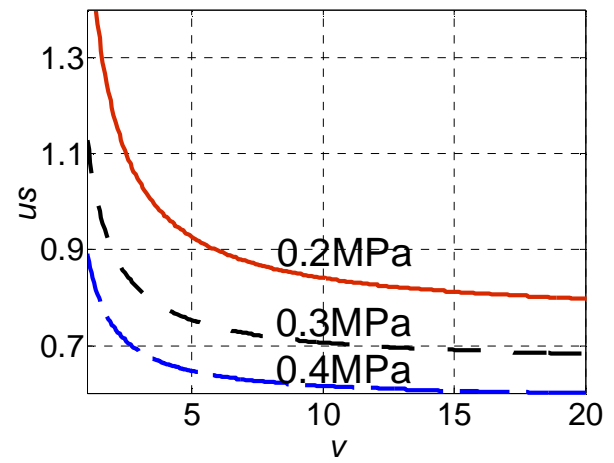

Fig.6 Asphalt friction coefficient at different pressure

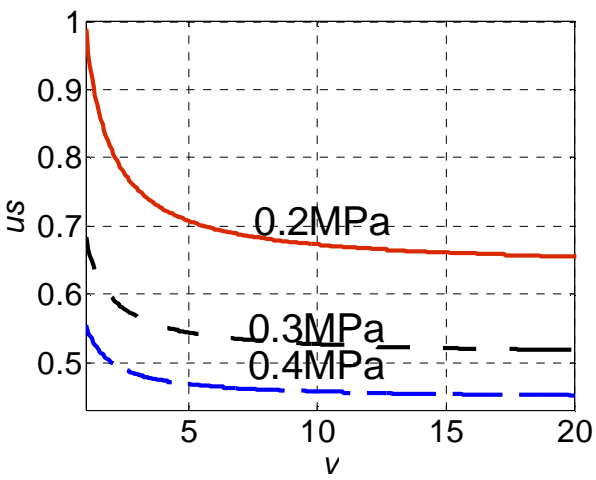

Fig.7 Granite friction coefficient at different pressure

Fig.6-Fig.7 show that, at the smaller contact pressure, the sliding friction coefficient is bigger; With the contact pressure increase the sliding friction coefficient decrease, and asphalt is higher than granite. At the smaller contact pressure, the sliding friction coefficient of asphalt has a 0.5 decrease with the slip velocity increase, the granite is 0.3 ;At the bigger contact pressure, the sliding friction coefficient of asphalt has a 0.3 decrease, the granite is 0.1 ; In the rang of $0.2 \sim 0.4 \mathrm{MPa}$, with the contact pressure increase the sliding friction coefficient of asphalt has a 0.3 decrease, the granite is 0.23 ; The degree of two kinds of road surface friction coefficient decreases reduce with contact pressure increases. At the smaller contact pressure, the influence of velocity is higher than at bigger contact pressure. Further more, when the velocity is higher than $10 \mathrm{~m} / \mathrm{s}$, the sliding friction coefficient of asphalt tends to a constant value, while to the granite the velocity value is $5 \mathrm{~m} / \mathrm{s}$.

The impact of different rubber and road character to friction coefficient. The sliding friction coefficient of different road for two kinds tread rubber was analyzed by simulation. Road affine parameter is shown in table 1, the simulation results are shown in fig. 8-fig.9.

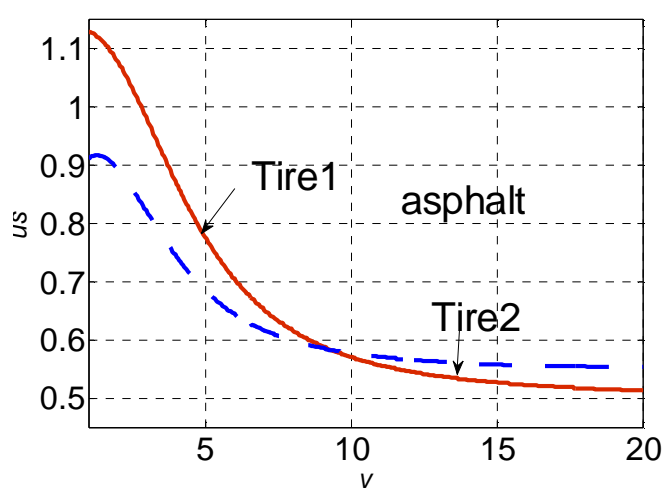

Fig.8 Friction coefficient of different tires on asphalt pavement

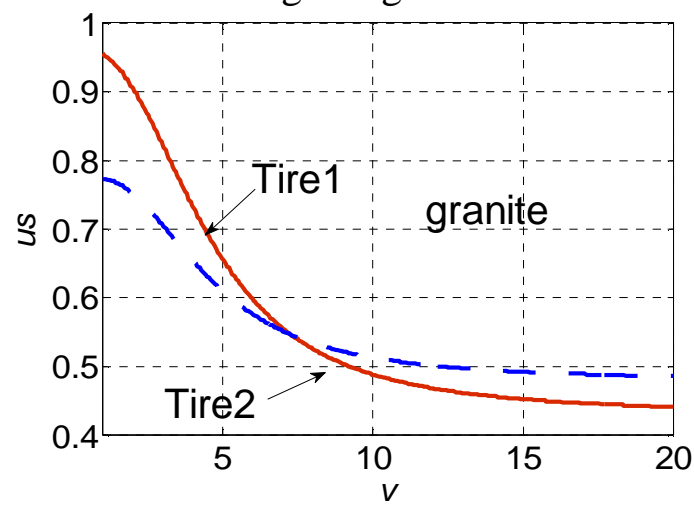

Fig.9 Friction coefficient of different tires on granite pavemen

It is shown as fig.8 and fig.9 that different tires and different road surfaces has the different sliding friction coefficient:

For asphalt pavement, at the same speed the excitation frequency is higher for the cut length of the vertical and horizontal are smaller, so the sliding friction coefficient is higher, the peak sliding friction coefficient of tire 1 is approximately 1.1 , tire 2 is 0.9 . Meanwhile at the lower speed, tire 1 has the higher sliding friction coefficient, at the higher speed tire 2 has the higher sliding friction coefficient.

For granite pavement, due to the cut-off length of the vertical is smaller and horizontal are bigger. So at the same speed the sliding friction coefficient is smaller, the peak sliding friction coefficient of tire 1 is approximately 0.95 , tire 2 is 0.8 . Meanwhile at the same speed, the sliding friction coefficient is 
also smaller than asphalt pavement. So it can see that, both tire and the road surface have impact to the sliding friction coefficient.

\section{Conlusion}

A improved sliding friction coefficient model is developed based on road surface character and road character to analyze how the road character, tire and contact pressure affect the sliding friction coefficient. Quantitatively evaluate the impact of contact pressure to the actual contact area. the impact of contact pressure, tire and road surface character to the sliding friction coefficient was analyzed. The simulation results show that the improved model can precisely analyze the impact of contact pressure and road surface character and tire to the sliding friction coefficient. This improved sliding friction coefficient model can also be used to analyze the tire dynamic friction character and to estimate the tire road friction force.

\section{Acknowledgements}

The authors wish to express their special thanks to the support of the Chinese National Natural Science Foundation(51078167) and the Transport Vehicle Safety Operation Technology Transportation Sector Key Laboratory Open Foundation (Vehicle Operating Risk Based on the Analysis of the Typical Traffic Accident ).

\section{References:}

[1] Olivier Le Maitre, Manfred S Ussner:SAE Paper 980256.

[2] Baumann F.W., Schreier H.H.and Simmermacher D.:SAE International Journal of Passenger Cars-Mechanical Systems, Vol.2 (2009) No. 01, p.333-343.

[3] Giuseppe Carbone:Journal of the Mechanics and Physics of Solids, Vol. 57 (2009) No.7, p.1093-1102.

[4] Gert Heinrich, Manfred Kluppel:Wear, Vol.265(2008) No.7,p.1052-1060.

[5] Ferry JD:Viscoelastic properties of polymers(New York:NY,Wiley,1980).

[6] G.Heinrich, M. Kluppel and T.A.Vilgis:Computational and Theoretical Polymer Science, Vol.10 (2000) No.1-2,p.53-61.

[7 ] Le Gal,L.Guy et:Wear. Vol.264 (2008) No.7,p.606-615.

[8] Savkoor A R:Wear, Vol.8(1965) No.3,p.222-237.

[9] S.Roux,J.Schmittbuhl,J.P.Vilotte and A.Hansen:Europhys Letters, Vol.23 (1993) ,p.277-282. 